\title{
Biologic Therapy for Moderate to Severe Psoriasis. Real-World Follow-up of Patients Who Initiated Biologic Therapy at Least 10 Years Ago
}

\author{
Laida Elberdín · Rosa M. Fernández-Torres · Sabela Paradela • \\ María Mateos • Eva Blanco • Vanesa Balboa-Barreiro • María I. Gómez-Besteiro • \\ Maria Outeda · Isabel Martín-Herranz • Eduardo Fonseca
}

Received: October 14, 2021 / Accepted: February 11, 2022 / Published online: February 28, 2022

(C) The Author(s) 2022

\section{ABSTRACT}

Introduction: The aim of this study was to evaluate response and drug survival of biologic therapy in patients with moderate to severe plaque-type psoriasis who initiated biologic therapy at least 10 years ago, in a real-world setting.

Methods: This was an observational retrospective follow-up study that included patients with moderate to severe plaque-type psoriasis who initiated biologic therapy between October 2006 and December 2009. Efficacy was

L. Elberdín $(\varangle) \cdot$ M. Mateos · M. Outeda .

I. Martín-Herranz

Department of Pharmacy, Complexo Hospitalario

Universitario de A Coruña (CHUAC), Sergas,

Instituto de Investigación Biomédica de A Coruña

(INIBIC), Universidade da Coruña (UDC), As Xubias,

84, 15006 A Coruña, Spain

e-mail: laida.elberdin@gmail.com

R. M. Fernández-Torres · S. Paradela · E. Blanco ·

E. Fonseca

Department of Dermatology, Complexo

Hospitalario Universitario de A Coruña (CHUAC),

Sergas,Instituto de Investigación Biomédica de A

Coruña (INIBIC), Universidade da Coruña (UDC), A

Coruña, Spain

V. Balboa-Barreiro · M. I. Gómez-Besteiro

Clinical Epidemiology and Biostatistics Unit, Complexo Hospitalario Universitario de A Coruña (CHUAC), Sergas, Instituto de Investigación

Biomédica de A Coruña (INIBIC), Universidade da

Coruña (UDC), A Coruña, Spain expressed as the percentage of patients achieving a 50, 75 and 90\% reduction from baseline in the Psoriasis Area and Severity Index (PASI 50, PASI 75, PASI 90, respectively) every 3 months during the first year of therapy and then every 12 months up to the end of follow-up or withdrawal from the study.

Results: A total of 56 patients were included in the study, representing 140 treatment lines (median 2, range 1-8); of these patients, 53 were still receiving biologic therapy at the end of the study. The mean duration of biologic therapy was 140.4 (range 47.6-175.4) months. Etanercept was used in $98.2 \%$ of patients, followed by efalizumab (42.9\%), adalimumab (41.1\%), ustekinumab (33.9\%) and infliximab (16.1\%). Treatment lines were switched in $62.1 \%$ of treatments: $24.3 \%$ due to secondary failure, $20.7 \%$ due to primary failure and $3.6 \%$ due to side effects. No patient treated with anti-interleukins had to discontinue treatment due to side effects. Ustekinumab had the highest drug survival.

Conclusions: This study in the real-world-setting shows maintenance of long-term efficacy and safety of biologic therapy in patients with moderate to severe plaque psoriasis in daily practice who initiated biologic therapy 10 years ago.

Keywords: Biologic therapy; Psoriasis; Realworld setting 


\section{Key Summary Points}

\section{Why carry out this study?}

There is a paucity of literature on longterm maintenance of the response to biologic therapy in psoriasis in a realworld setting.

This study evaluates patient response to biologic therapy based on sequential Psoriasis Area and Severity Index (PASI) scores instead of assessing efficacy in terms of PASI 75 response.

\section{What was learned from the study?}

The results further our understanding of the behavior of biologic therapies in the real-life setting.

This study provides dermatologists with current evidence on and a summary of patient response to and drug survival of biologic therapies in patients with moderate to severe plaque-type psoriasis.

\section{INTRODUCTION}

The efficacy and safety profiles of biologic therapies in psoriasis have been studied in numerous clinical trials. However, it should be noted that in most of these trials, efficacy was assessed based on the percentage of patients who achieved PASI 75. Also, clinical experience in the treatment of psoriasis with biologic therapies has shown relevant variations between the results of clinical trials and those obtained in daily practice.

The first biologic therapy authorized for moderate-to-severe plaque-type psoriasis in Europe was efalizumab, in October 2004. In February 2009, the European Medicines Agency suspended the marketing authorization for efalizumab after three confirmed cases of progressive multifocal leukoencephalopathy were reported [1].
Maintaining response to treatment is important in chronic conditions that require long-term treatment, such as psoriasis. It is known that drug survival decreases over time for all biologic therapies, mainly due to lack/ loss of efficacy, eventual remission and side effects, depending on the study. Drug survival of biologics, defined as the time from initiation to discontinuation of a particular treatment, is well documented in several registries and cohort studies [2-4].

Only a limited number of studies have considered long-term maintenance of the response as an indicator of treatment efficacy $[5,6]$. Psoriasis response data to biologic therapy in clinical practice are scarce, such as studies evaluating long-term efficacy [7].

In the study reported here we have evaluated the response to biologic therapies, based on sequential PASI scores, as well as drug survival in patients with moderate to severe plaque-type psoriasis who initiated biologic therapy at least 10 years ago, in a real-world setting.

\section{METHODS}

This is an observational retrospective follow-up study in patients with moderate to severe plaque type psoriasis who initiated biologic therapy between October 2006 and December 2009. Eligible patients could have started treatment with efalizumab (withdrawn in February 2009), etanercept, adalimumab, infliximab or ustekinumab, which were the biological treatments available at that time. The choice of biologic was made according to the availability of the drug at the time of prescribing and the characteristics of the patients. During patient recruitment, the biologics available for treatment were efalizumab, infliximab, etanercept and adalimumab. Efalizumab was authorized for moderate to severe plaque psoriasis in Spain in October 2004, infliximab in November 2005, etanercept in April 2007, adalimumab in May 2008 and ustekinumab in February 2009. Efalizumab was the drug of first choice until it was withdrawn from the drug market. Once etanercept became available, it was the biologic of second choice after treatment failure with 
efalizumab. When the marketing of efalizumab was discontinued, those patients treated with this drug were switched to etanercept, which became the first-choice biologic medicine for naïve patients. Treatment with infliximab requires intravenous infusion and, consequently, infliximab was reserved for severe patients who had associated comorbidities for whom efalizumab or etanercept were not suitable. Adalimumab and ustekinumab were marketed later than the others, and were therefore used in patients who had failed first-line treatment.

All patients enrolled in the study were followed up at the Department of Dermatology of the University Hospital of La Coruña (Spain) from October 2006 to December 2019. All patients received the standard doses established in the technical data sheet of the drugs. PASI and Body Surface Area (BSA) responses were evaluated at the first month and then every 3 months during the first year of treatment, and then every 12 months until the end of the follow-up or until the withdrawal of biologic therapy due to poor tolerance, lack of efficacy or adverse events.

The response to biologic therapy during the study period was evaluated in comparison with the baseline Psoriasis Area and Severity Index (PASI) score before initiation of a biologic therapy (course baseline). In addition, efficacy was expressed as the percentage of patients achieving a 50, 75 and $90 \%$ reduction from baseline in the PASI score (PASI 50, PASI 75, PASI 90, respectively) $[5,6]$.

Failure on biologic therapies was categorized into primary failure, secondary failure and side effects. Primary failure was defined as an insufficient response (patients not achieving PASI 50) at week 12-16, according to the medication. Secondary failure was defined as the loss of response (PASI $>50 \%$ of initial value) in a patient who had previously achieved PASI 50 response at week 12-16 [8].

\section{Inclusion and Exclusion criteria}

Patients were eligible for inclusion in the study if they satisfied the following criteria: (1) aged $\geq$
18 years; (2) had with moderate to severe plaque psoriasis and initiated treatment with biologic drugs for psoriasis at least 10 years previously; (3) had with PASI or BSA scores of $\geq 10$ or had received systemic treatment for their psoriasis at study inclusion.

The exclusion criteria were: (1) presence of erythrodermic, guttate or pustular psoriasis; (2)evidence of other skin conditions that would interfere with the evaluation of psoriasis.

\section{Ethics and Authorizations}

This study was approved by the Ethic Committee for Clinical Investigation of Galicia (Protocol Code 2017/378) and classified as a post authorization prospective study by the Agencia Española del Medicamento y Productos Sanitarios (Protocol code EFP-FAR-2017-01). Prior to inclusion into the study, each patient signed a written informed consent form. This study was conducted in accordance with the Helsinki Declaration of 1964 and its later amendments.

\section{Statistics}

A descriptive analysis of all variables included in the study was performed. Quantitative variables were expressed as the mean \pm standard deviation (SD), or as the median and range. Qualitative variables were expressed as an absolute value $(n)$ and percentage. Group differences were compared using the Pearson Chi-square $\left(\chi^{2}\right)$ or Fisher's exact test for categorical variables. The Kaplan-Meier survival analysis model was used to estimate drug survival, with treatment line considered as the unit of analysis. The hazard ratio (HR) with $95 \%$ confidence intervals (CIs) were reported. A $p$ value $<0.05$ was considered to be statistically significant.

The statistical analysis was carried out using the SPSS version 24.0 statistical program (IBM SPSS, Armonk, NY, USA). 


\section{RESULTS}

\section{Demographic and Treatment Characteristics}

All patients with moderate to severe psoriasis treated at our hospital who met the inclusion criteria were included in the study: 56 patients and 140 treatment lines. Of these 56 patients, $23.2 \%$ had psoriatic arthritis, with mainly dermatological symptoms. The median duration of biologic therapy was 140.4 (range 47.6-175.4) months; 54 patients had received biologic therapy for $>10$ years. The median number treatment lines was two (range 1-8). No concomitant systemic anti-psoriatic therapy was used during biologic therapy at the efficacy evaluation time points. The demographic and clinical characteristics of the study population are summarized in Table 1 . At the end of the study, 53 of the 56 patients were still undergoing treatment. Of the three patients who had suspended biologic therapy, one died of causes unrelated to psoriasis or the biologic treatment, one stopped due to a wish to become pregnant and the third stopped due to urinary tract infection during etanercept treatment. Ten patients received only one line of biologic treatment; all of these patients were treated with etanercept and all were still receiving this biologic at the end of the study period.

Etanercept was the most used biologic (98.2\% of patients, $39.3 \%$ of treatments), followed by efalizumab (42.9\% of patients, $17.1 \%$ of treatments) adalimumab (41.1\% of patients, $16.4 \%$ of treatments), ustekinumab (33.9\% of patients, $13.6 \%$ of treatments), infliximab (16.1\% of patients, $6.4 \%$ of treatments) and secukinumab $(7.1 \%$ of patients, $4.3 \%$ of treatments). Treatment characteristics of the study population for treatment lines 1 to 5 are shown in Table 2.

Etanercept was the most widely used biologic medicine in the first- $(53.6 \%$ of patients) and second-line $(52.2 \%$ of patients) treatments. Of the 55 patients who started treatment with etanercept, $41.8 \%$ were still continuing treatment with this biologic at the end of the study period.
Table 1 Demographic and clinical characteristics of the study population

\begin{tabular}{ll}
\hline $\begin{array}{l}\text { Demographic and clinical } \\
\text { characteristics }\end{array}$ & Values \\
\hline Total number of patients & $56(100.0 \%)$ \\
Gender & \\
Male & $42(75.0 \%)$ \\
Female & $14(25.0 \%)$ \\
Diagnosis & \\
Psoriasis & $43(76.8 \%)$ \\
Psoriatic arthritis & $13(23.2 \%)$ \\
Age at diagnosis (years) & $25.5(4.0-60.0)$ \\
Biologic treatment & \\
PASI score baseline & $10.0(1.8-50.0)$ \\
Duration (months) & 140.4 \\
& $(47.6-175.4)$ \\
Treatment lines $(n)$ & $2(1-8)$ \\
1 treatment line & $10(17.8 \%)$ \\
2 treatment lines & $24(42.9 \%)$ \\
3 treatment lines & $12(21.4 \%)$ \\
4 treatment lines & $7(12.5 \%)$ \\
5 treatment lines & $2(3.6 \%)$ \\
6 treatment lines & $0(0.0 \%)$ \\
7 treatment lines & $0(0.0 \%)$ \\
8 treatment lines & $1(1.8 \%)$ \\
\hline
\end{tabular}

Values are given as the number $(\mathrm{n})$ with the percentage in parentheses, or as the median with the range in parentheses, as appropriate

PASI Psoriasis Area and Severity Index

Efalizumab was only prescribed as a first-line treatment. Following its withdrawal from the drug market, the 24 patients on this biologic switched to etanercept; of these, 12 (50.0\%) were still receiving etanercept treatment at the end of the study period.

The frequency of use of biologic drugs in the first to fifth lines of treatment and other 
Table 2 Treatment characteristics of the total study population and the first to fifth treatment lines

\begin{tabular}{|c|c|c|c|c|c|c|}
\hline $\begin{array}{l}\text { Treatment } \\
\text { characteristics }\end{array}$ & $\begin{array}{l}\text { Total } \\
\text { population }\end{array}$ & $\begin{array}{l}\text { First line of } \\
\text { treatment }\end{array}$ & $\begin{array}{l}\text { Second line } \\
\text { line of } \\
\text { treatment }\end{array}$ & $\begin{array}{l}\text { Third line } \\
\text { line of } \\
\text { treatment }\end{array}$ & $\begin{array}{l}\text { Fourth line } \\
\text { line of } \\
\text { treatment }\end{array}$ & $\begin{array}{l}\text { Fifth line } \\
\text { line of } \\
\text { treatment }\end{array}$ \\
\hline $\begin{array}{l}\text { Number of patients, } \\
n(\%)\end{array}$ & $56(100.0)$ & $56(100.0)$ & $46(82.1)$ & $22(39.3)$ & $10(17.9)$ & $3(5.4)$ \\
\hline \multicolumn{7}{|c|}{ Biologic treatment, median (range) } \\
\hline PASI initial score & $\begin{array}{l}10.0 \\
(1.8-50.0)\end{array}$ & $\begin{array}{l}10.0 \\
(1.8-50.0)\end{array}$ & $6.7(0-32.4)$ & $\begin{array}{l}10.4 \\
(1.8-34.3)\end{array}$ & $15.5(0-26.6)$ & $\begin{array}{l}12.6 \\
\quad(10.6-14.4)\end{array}$ \\
\hline Duration (months) & $\begin{array}{l}140.4 \\
\quad(47.6-175.4)\end{array}$ & $\begin{array}{l}20.5 \\
\quad(1.2-152.6)\end{array}$ & $\begin{array}{l}78.1 \\
\quad(3.0-155.6)\end{array}$ & $\begin{array}{l}22.7 \\
\quad(3.3-123.7)\end{array}$ & $\begin{array}{l}54.0 \\
\quad(12.8-115.8)\end{array}$ & $8(6.1-84.9)$ \\
\hline \multicolumn{7}{|c|}{ Biological medicines, $n$ (\%) } \\
\hline Etanercept & $55(98.2)$ & $30(53.6)$ & $24(52.2)$ & $1(4.5)$ & - & - \\
\hline Efalizumab & $24(42.9)$ & $24(42.9)$ & - & - & - & - \\
\hline Infliximab & $9(16.1)$ & $2(3.6)$ & - & $4(18.2)$ & $3(30.0)$ & - \\
\hline Adalimumab & $23(41.1)$ & - & $12(26.1)$ & $10(45.5)$ & $1(10.0)$ & - \\
\hline Ustekinumab & $19(33.9)$ & - & $10(21.7)$ & $5(22.7)$ & $3(30.0)$ & $1(33.3)$ \\
\hline Secukinumab & $4(7.1)$ & - & - & $1(4.5)$ & $2(20.0)$ & $1(33.3)$ \\
\hline Ixekizumab & $1(1.8)$ & - & - & - & $1(10.0)$ & $1(33.3)$ \\
\hline Apremilast & $1(1.8)$ & - & - & $1(4.5)$ & - & - \\
\hline $\begin{array}{l}\text { Reason for } \\
\text { discontinuation of } \\
\text { series of treatment, } \\
n(\%)\end{array}$ & $140(100.0)$ & $46(82.1)$ & $23(50.0)$ & $11(50.0)$ & $4(36.4)$ & $1(33.3)$ \\
\hline Total & $87(62.1)$ & & & & & \\
\hline Primary failure & $29(20.7)$ & $15(32.6)$ & $7(30.5)$ & $4(36.3)$ & - & $1(100)$ \\
\hline Secondary failure & $34(24.3)$ & $15(32.6)$ & $13(56.6)$ & $3(27.3)$ & $3(75.0)$ & - \\
\hline Contraindication & $4(2.9)$ & $2(4.4)$ & $1(4.3)$ & $1(9.1)$ & - & - \\
\hline Side effects & $5(3.6)$ & $2(4.4)$ & $1(4.3)$ & $2(18.2)$ & - & - \\
\hline Others & $15(10.7)$ & $12(26.0)$ & $1(4.3)$ & $1(9.1)$ & $1(25.0)$ & - \\
\hline
\end{tabular}

characteristics are shown in Table 2. More than five lines of treatment was required by only one patient, who needed eight treatment lines. This patients received treatment with apremilast and ixekizumab in the sixth and seventh lines, respectively, both jof which were suspended due to primary failure. At the end of the study period, the patient was continuing treatment with guselkumab in the eighth line of treatment. 


\section{Reasons for Switching}

A total of 140 treatment lines were associated with the 56 patients enrolled in the study. Analysis revealed that the mainly reasons to switch of biologic treatment were secondary $(24.3 \%)$ and primary $(20.7 \%)$ failures. In the first line of treatment, the withdrawal of efalizumab was also one of the main causes for switching biologic (26.0\%) (Table 2).

Four patients $(7.1 \%)$ switched treatments due to the development of contraindications for some biologic therapies. Two patients were diagnosed with heart failure during treatment with etanercept at first-line treatment; both patients were switched to ustekinumab. One patient receiving ustekinumab as second-line treatment stopped using this biologic because of suspected myelodysplastic syndrome; treatment was switched to apremilast. One patient receiving infliximab as third-line treatment was diagnosed of breast cancer; biologic therapy was temporarily stopped and restarted 7 years later with secukinumab.

Five $(8.9 \%)$ patients discontinued their treatment due to adverse events: all were treated with anti-tumor necrosis factor (TNF). No patient treated with anti-interleukins had to discontinue treatment due to side effects. In a first-line treatment with etanercept, one patient developed generalized itching; this patient was switched to ustekinumab at the 36th month of treatment, with good subsequent control. One patient receiving infliximab as first-line treatment developed lichen planopilaris at the 24th month of treatment; this patient was switched to ustekinumab. Another patient was diagnosed with urinary tract infection during the 40th month of second-line treatment with etanercept; this patient discontinued biologic therapy was still without treatment at the end of the study period. A patient treated with infliximab as third-line treatment was diagnosed with severe thrombocytopenia and macrocytic anemia at the ninth month of treatment, and was switched to ustekinumab. Another patient suffered generalized itching during infliximab treatment that was suspended after 8 months. Four years later this patient was treated with adalimumab (because no more biologics were available at that moment) and then switched to secukinumab after 1 year because of secondary failure and the development of pruritus once again.

Treatment characteristics of the study population in each line are summarized in Table 2.

\section{Efficacy Measures}

Analysis of treatment response in treatment groups with $\geq 10$ patients revealed that regarding first-line treatments, $73.3 \%$ and $50.0 \%$ of patients who received treatment with etanercept and $54.2 \%$ and $33.3 \%$ of those receiving efalizumab achieved PASI 75 and PASI 90 response, respectively. There were no significant differences between response to biologics in first-line treatments $(p>0.05)$.

In second-line treatments, $91.7 \%$ and $79.2 \%$ of patients treated with etanercept, $75.0 \%$ and $66.7 \%$ of those treatmed with adalimumab and $90.0 \%$ and $90.0 \%$ of those treated with ustekinumab achieved PASI 75 and PASI 90 response, respectively. There were no significant differences in the response to etanercept between the first- and second-line treatments $(p>0.05)$.

In third-line treatments, $100.0 \%$ and $90.0 \%$ of patients treated with adalimumab achieved PASI 75 and PASI 90 response, respectively.

Of the three patients who received five lines of treatment, two were continuing treatment at the end of the study with a good response (PASI 90), after 6 and 85 months of treatment, respectively. The third patient did not reach PASI 50 at 9, 4 and 6 months of treatment, and therefore needed to switch to apremilast, ixekizumab and guselkumab successively. At the end of the study, this patient was continuing treatment with guselkumab with good response (PASI90) at 10 months.

\section{Drug Survival}

The 140 treatment lines of the 56 patients were evaluated. Ustekinumab had the highest drug survival. Taking ustekinumab as the reference drug, we observed that patients being treated with efalizumab (HR 7.94, 95\% CI 3.42-18.44; $p<0.001$ ) and infliximab (HR 2.79, 95\% CI 


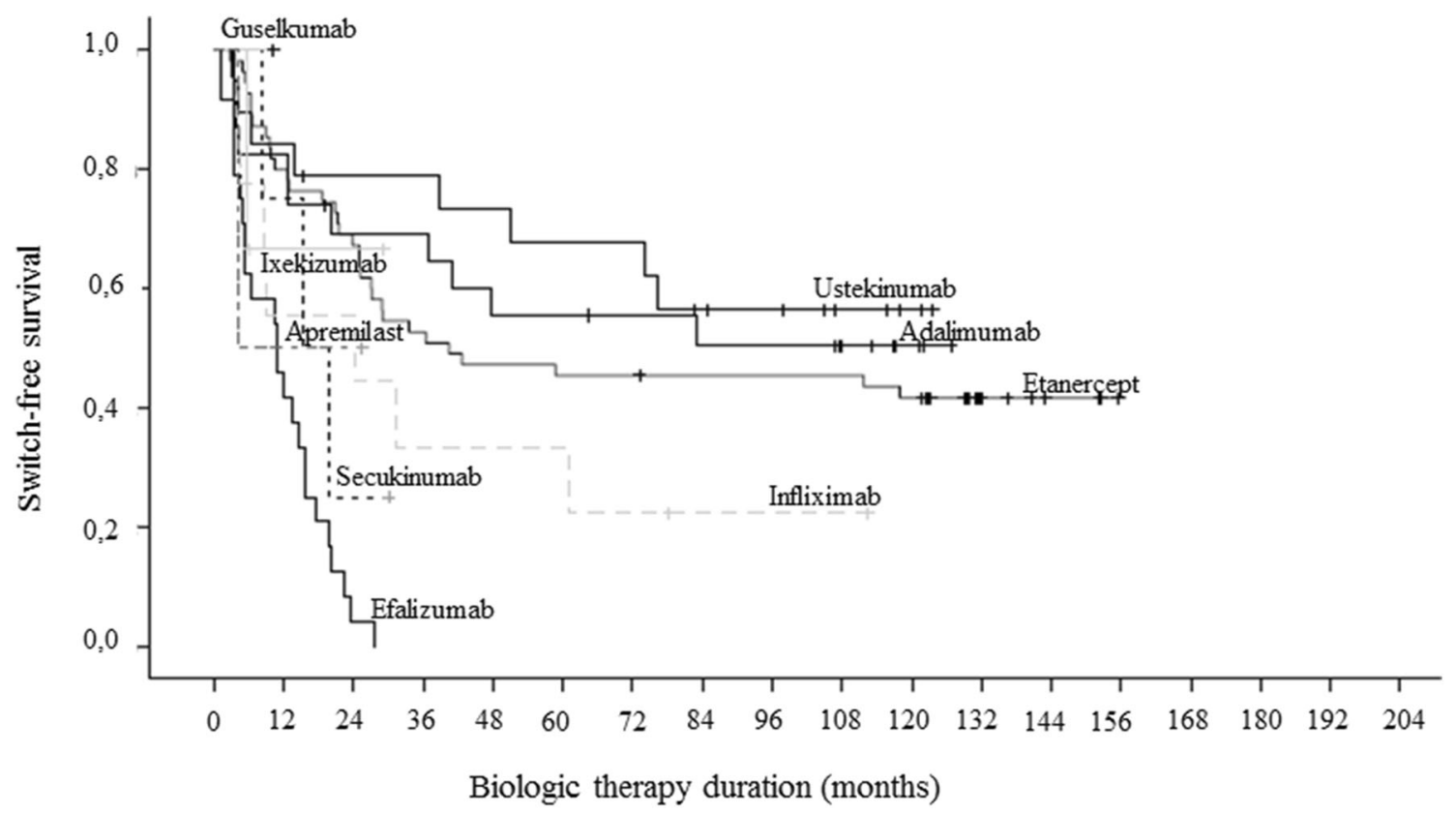

$\begin{array}{lll} & \text { HR }(\mathbf{9 5 \%} \text { CI) } & \text { p-value } \\ \text { Efalizumab } & 7.94(3.42-18.44) & 0.000^{*} \\ \text { Secukinumab } & 3.67(0.96-14.11) & 0.058 \\ \text { Infliximab } & 2.79(1.01-7.72) & 0.048^{*} \\ \text { Ixekizumab } & 2.04(0.25-16.45) & 0.505 \\ \text { Etanercept } & 1.51(0.69-3.28) & 0.299 \\ \text { Adalimumab } & 1.25(0.50-3.11) & 0.631 \\ \text { Ustekinumab } & 1.00 \text { (Reference category) } & \end{array}$

Fig. 1 Drug survival of each biologic (Kaplan-Meier survival curves for biologic drugs). Asterisk indicates significant difference at $p<0.05$. CI Confidence interval, $H R$ hazard ratio

$1.01-7.72 ; p=0.048$ ) showed a trend of significantly higher HR of switching. We also observed that the switch rate of other biologics did not significantly differ from that of ustekinumab. The results are shown in Fig. 1.

\section{DISCUSSION}

This observational retrospective follow-up study, based on 56 patients, describes a realworld use of biologic therapy in patients with moderate to severe plaque-type psoriasis who initiated biologic therapy at least 10 years ago. This report provides valuable insight into the efficacy and safety of biologic therapy in clinical practice for up to 10 years of continued use.

Most patients continued biologic therapy at the end of the study period with good response, a duration of treatment of $>10$ years and a median of two treatment lines (range 1-8). The efficacy and safety of biologic therapy have been demonstrated in several clinical trials in a short period of time. However, studies evaluating the continued use of biologic therapy for $>$ 10 years in the real-world setting are scarce $[3,9]$.

In a systematic review of drug survival in biologic treatments in psoriasis, No et al. [3] selected 36 studies in which the inclusion criteria of patients previously treated with 
biologics varied widely and included patients naïve to biologics, patients who had failed biologic treatment and patients whose exposure to biologics was unknown. The majority of biologic treatments were with etanercept (43.1\%), followed by adalimumab (25.6\%), ustekinumab (22.6\%) and infliximab (8.7\%). In our study, the most prescribed biologic was also etanercept: $39.3 \%$ of treatments in $98.2 \%$ of patients; efalizumab was the second most commonly prescribed biologic (in $17.1 \%$ of treatments), followed by adalimumab (16.4\%), ustekinumab $(13.6 \%)$ and infliximab (6.4\%). Thus, the use of biologics in terms of most prescribed drugs in our study is in agreement with the order in the study of No et al. [3], with the exception of efalizumab. In our study, efalizumab was only prescribed as first-line biologic therapy due to its withdrawal from the drug market in 2009. The 24 patients who were being treated with efalizumab as first-line treatment at that time were switched to etanercept.

The median number of treatment lines in our study was two (range 1-8). We cannot compare these data with data from other studies since the latter only distinguish between naive and non-naive patients $[3,9]$.

Roche et al. [7] describe drug survival in a 12-year, long-term real-life experience with biologics in psoriasis patients. In their study, patients received a mean $( \pm \mathrm{SD})$ of $2.1 \pm 0.9$ different biologics, which is similar to the number of biologics seen in our study. However, Roche et al. included all patients treated with biologics during the 12-year study period, did not define a minimum treatment time and excluded patients treated with efalizumab; in comparison, in our study, we only included patients that initiated biologic therapy at least 10 years ago.

In our study, lack of efficacy of a specific biologic was the most common reason to discontinue that specific biologic therapy, which is in agreement with published studies [2, 3, 7, 9]. The precise mechanism that causes the clinical decline is not entirely understood, but may be associated with immune-mediated mechanisms by which antidrug antibodies are developed via a T-cell-dependent humoral response. These anti-drug antibodies likely form immune complexes that interrupt drug-end target interaction or increase drug clearance, thereby altering the efficacy and bioavailability of biologic drugs [3]. These data arenot available in our study because this strategy was not used in our hospital until 2013, 7 years after the start of the study inclusion period.

The percentage of discontinuation of an individual drug in our study was $62.1 \%$, which is very similar to the $65.4 \%$ found by Roche et al. [7]. However, these authors reported a loss efficacy percentage of $44.9 \%$ and serious adverse events in $17.4 \%$ of patients; these values were $24.3 \%$ and $3.6 \%$ in our study, respectively.

Only five of our patients discontinued a biologic due to adverse events, all were treated with an anti-TNF drug. The adverse effects resolved upon discontinuation of the specific biologic or switch to an anti-TNF drug. No patients treated with anti-interleukins had to discontinue their treatment due to side effects. This result supports the long-term safety of biologic therapy.

We found that ustekinumab had the best drug survival in psoriatic patients who initiated biologic therapy at least 10 years ago. This result is consistent with those of previous studies on drug survival [2-4, 7, 9-11].

In addition, we found that patients being treated with efalizumab and infliximab showed a trend towards a significantly higher HR of switching than those receiving ustekinumab, but this trend was not observed with the other biologics. The reason for the higher probability of switching among patients receiving efalizumab could be related to the suspension of the marketing authorization for efalizumab in February 2009, which resulted in patients having to change treatment at that time [1]. Regarding infliximab, the higher probability of switching seen among patients receiving this biologic may be due to its intravenous administration route. Currently, there are several biologic treatment options administered subcutaneously that patients are more comfortable with. However, we did not have adequate data on the drug survival of biologics that are newly available on the market, such as secukinumab, ixekizumab or guselkumab, similar to the Lin et al. study [9]. 
Puig et al. [2] describe the drug survival of conventional and biologic therapies in patients with moderate to severe psoriasis in a real-world setting. Nevertheless, their study is a 2-year observational prospective study that only evaluates the first-line treatments. Drug survival decreases over time for all conventional and biologic therapies, mainly due to lack/loss of efficacy, eventual remission and side effects, depending on the study [2].

The limitations of our study are its singlecenter retrospective design, the limited number of patients included and its analysis of the realworld environment in which the data may be influenced by different times at which biologics are introduced onto the market.

\section{CONCLUSIONS}

This real world-setting study shows maintenance of long-term efficacy and safety of biologic therapy in patients with moderate to severe plaque psoriasis who initiated biologic therapy at least 10 years ago. Long-term clinical practice studies of biological psoriasis treatments are essential to understand the behavior of these drugs in real life, outside the context of clinical trials.

\section{ACKNOWLEDGEMENTS}

Funding. The journal's Rapid Service Fee was paid for by Fundación Profesor Novoa Santos (A Coruña. Spain).

Authorship. All named authors meet the International Committee of Medical Journal Editors (ICMJE) criteria for authorship for this article, take responsibility for the integrity of the work as a whole, and have given their approval for this version to be published.

Author Contributions. All authors contributed to the study conception and design. Material preparation, data collection and analysis were performed by Laida Elberdín. The first draft of the manuscript was written by Laida
Elberdín and all authors commented on previous versions of the manuscript. All authors read and approved the final manuscript.

Disclosures. Laida Elberdín has participated in continuing medical education events supported by unrestricted educational grants from Abbvie, Lilly, Novartis, Pfizer and Shire. Rosa M Fernández-Torres has participated in clinical trials sponsored by companies that manufacture drugs used for the treatment of psoriasis and in continuing medical education events supported by unrestricted educational grants from Abbvie, Celgene, Janssen-Cilag, Leo, MSD, Novartis and Pfizer. Sabela Paradela and Eduardo Fonseca have participated in clinical trials sponsored by companies that manufacture drugs used for the treatment of psoriasis and in continuing medical education events supported by unrestricted educational grants from Abbvie, Almirall, Celgene, Janssen-Cilag, Leo, MSD, Novartis and Pfizer. Isabel Martín has participated in continuing medical education events supported by unrestricted educational grants from Abbvie, Janssen-Cilag, Novartis, Pfizer, Sanofi and Roche. María Mateos, Eva Blanco, Vanesa Balboa-Barreiro, María Inmaculada Gómez-Besteiro and María Outeda declare that they have no conflict of interest.

Compliance with Ethics Guidelines. This study was approved by the Ethic Committee for Clinical Investigation of Galicia (Protocol Code 2017/378) and classified as a post authorization prospective study by the Agencia Española del Medicamento y Productos Sanitarios (Protocol code EFP-FAR-2017-01). Prior to inclusion into the study, each patient signed a written informed consent form. This study was conducted in accordance with the Helsinki Declaration of 1964 and its later amendments.

Data Availability. All data generated or analyzed during this study are included in this published article.

Open Access. This article is licensed under a Creative Commons Attribution-NonCommercial 4.0 International License, which permits any non-commercial use, sharing, adaptation, 
distribution and reproduction in any medium or format, as long as you give appropriate credit to the original author(s) and the source, provide a link to the Creative Commons licence, and indicate if changes were made. The images or other third party material in this article are included in the article's Creative Commons licence, unless indicated otherwise in a credit line to the material. If material is not included in the article's Creative Commons licence and your intended use is not permitted by statutory regulation or exceeds the permitted use, you will need to obtain permission directly from the copyright holder. To view a copy of this licence, visit http://creativecommons.org/licenses/by$\mathrm{nc} / 4.0 /$.

\section{REFERENCES}

1. Baniandrés O, Pulido A, Silvente C, Suárez R, Lázaro P. Evolución clínica de los pacientes psoriásicos tratados con efalizumab al suspender el fármaco. Actas Dermosifiliogr. 2010;101:423-9.

2. Puig L, Carrascosa JM, Daudén E, Sulleiro S, Guisado C. Drug survival of conventional systemic and biologic therapies for moderate-to-severe psoriasis in clinical practice in Spain: prospective results from the SAHARA study. J Dermatolog Treat. 2020;31:344-51.

3. No DJ, Inkeles MS, Amin M, Wu JJ. Drug survival of biologic treatments in psoriasis: a systematic review. J Dermatolog Treat. 2018;29:460-6.

4. Menter A, Papp KA, Gooderham M, et al. Drug survival of biologic therapy in a large, disease-based registry of patients with psoriasis: results from the Psoriasis Longitudinal Assessment and Registry (PSOLAR). J Eur Acad Dermatol Venereol. 2016;30: 1148-58.

5. Elberdín L, Fernández-Torres R, Paradela S, et al. Ustekinumab treatment for moderate to severe psoriasis. Eight-year real-world follow-up of 61 cases in a tertiary level hospital. J Dermatolog Treat. 2019;31(7):698-701.

6. Fernández-Torres RM, Paradela S, Fonseca E. Longterm response to etanercept monotherapy in moderate to severe psoriasis: Assessment in daily practice by the maintenance of low values of PASI and BSA. J Dermatolog Treat. 2014;25:54-6.

7. Roche H, Bouiller K, Puzenat E, et al. Efficacy and survival of biologic agents in psoriasis: a practical real-life 12-year experience in a French dermatology department. J Dermatolog Treat. 2019;30:540-4.

8. Fonseca E, Iglesias R, Paradela S, Fernández-Torres RM, Elberdín L. Efficacy and safety of adalimumab in psoriatic patients previously treated with etanercept in a real-world setting. J Dermatolog Treat. $2015 ; 26(3): 217-22$.

9. Lin P, Wang S, Chi C. Drug survival of biologics in treating psoriasis: a meta-analysis of real- world evidence. Sci Rep. Sci Rep. 2018;8(1):1606.

10. Marinas JEC, Kim WB, Shahbaz A, Qiang JK, Greaves S. Survival rates of biological therapies for psoriasis treatment in real-world clinical practice: a Canadian multicentre retrospective study data collection. Australas J Dermatol. 2018;59:e11-4.

11. Cozzani E, Wei Y, Burlando M, Signori A, Parodi A. Serial biologic therapies in psoriasis patients: a 12-year, single-center, retrospective observational study. J Am Acad Dermatol. 2020;82:37-44. 